\title{
Prognostic scores in heart failure patients
}

\author{
Iwona Cygankiewicz \\ Department of Electrocardiology, Medical University of Lodz, Poland
}

\section{Article p. 578}

Heart failure (HF), an epidemic of the XXI century, is a leading cause of hospitalizations and mortality in developed countries. Recent data indicate that $1-2 \%$ of an adult population suffers from HF, with the prevalence being as high as $10 \%$ in elderly. With over 500,000 new cases recognized yearly in the US, admissions for "de novo" HF and readmissions for pump failure deterioration constitute one of the leading causes of hospitalizations. Despite advancement in modern treatment, and some improvement in survival observed during last decade, the diagnosis of HF carries a very poor prognosis for patients, and implicates substantial financial impact on healthcare system [1-3]. Recognition of $\mathrm{HF}$ implicates management strategies including pharmacotherapy, electrotherapy and lifestyle change. These treatment strategies are aimed to decrease symptoms, and number of readmissions for HF worsening, prevent life-threatening arrhythmias, and improve quality of life. Recent years experienced an increasing interest in home monitoring, self-care and family support that are crucial for achieving abovementioned therapeutic goals $[4,5]$. Efforts of the investigators, and clinicians are focused on the optimal risk stratification of patients who require more intensive treatment strategies, and better selection of those in whom implanted devices may prolong life $[1,2,6,7]$.

Stratification of risk in patients with HF is based on plethora of clinical, laboratory and imaging tests representing factors contributing to progression of a disease. Advanced age, coexisting comorbidities like diabetes, renal dysfunction or atrial fibrillation are the most significant covariates influencing survival in HF patients [1, 2, 6, 8]. However, taking into account multitude of factors con- tributing to $\mathrm{HF}$ progression it is unlikely than one sole variable may indicate patients at poor prognosis. Therefore, attempts are being made to create multivariate risk models that will provide better estimation of individual patient's risk. To facilitate the clinical use of these models several risk scores based on the results of multivariate analyses have been created to predict mortality and HF progression [8-12].

The Seattle Heart Failure Model is the most frequently used in clinical practice risk calculator based on multi-parameter assessment for estimation of 1-, 2- and 5-year mortality in HF patients. This risk model was derived in a population of 1125 $\mathrm{HF}$ patients and validated in 5 other HF cohorts and is based on simple clinical, pharmacological, laboratory data and presence of an implanted device [9]. The risk calculator is available online (www.seattleheartfailuremodel.org) and then it is frequently used in clinical practice. Nevertheless, this risk score, published in 2006 is based on patients with predominantly left ventricular (LV) systolic HF. Recent years have experienced a significant improvement in management of acute coronary syndromes and consequently brought the change in the epidemiology of HF population with an increasing number of patients with preserved LV function. Even though it was documented that this subpopulation carries similar risk of mortality as typical systolic dysfunction, few studies addressed the problem of complex risk stratification in these patients $[10,13,14]$. The Spanish MUSIC study enrolled between 2003 and 2004 nearly 1000 ambulatory patients with mild to moderate (II-III NYHA class) HF independently on etiology, including patients with either depressed or preserved LV function. The authors of this study developed a risk score based on 10 clinical, echocardiographic, ECG and laboratory variables that were independently associated with worse survival [10].

Address for correspondence: Iwona Cygankiewicz, MD, PhD, Department of Electrocardiology, Medical University of Lodz, ul. Sterlinga 1/3, 91-425 Lodz, Poland, tel/fax: +48 42 6644269/+48 42 6644304, e-mail: cygankiewicz@interia.pl 
Separate risk scores were developed to predict allcause mortality, cardiac death, sudden death or HF progression. The prediction risk scores were tested in the total MUSIC population as well as in subgroups of patients with preserved LV ejection fraction ( $>45 \%)$ which accounted for $25 \%$ of the total population, and showed similar results in patients with preserved and depressed LV function. Most of risk prediction scores evaluate long term survival; however the ability to predict survival up to one year of follow up is gaining more and more attention as current guidelines for ICD/CRT-D implantation recommend that the ability to survive in a good functional status should be considered as one of the selection criteria for a device implantation $[1,7]$. Several scores tested to predict one year survival in ICD/CRT recipients put emphasis on importance of comorbidities, especially renal dysfunction in predicting survival $[8,9,11,12]$. The study by Goldenberg at al. [12] showed that a bedside clinical risk score composed from 5 variables (NYHA functional class $>$ II, age $>70$ years, blood urea nitrogen $>26 \mathrm{mg} / \mathrm{dL}$, QRS duration $>120 \mathrm{~ms}$, and atrial fibrillation) was able to identify patients who are "too sick" or "too healthy" to benefit from ICD implantation.

The study by Gomez de la Camara et al. [15], published in the current issue of "Cardiology Journal", follows the line of research in field of better stratification of patients with $\mathrm{HF}$, and confirms previously published results that underlined age, and renal dysfunction, hyponatremia, and systolic dysfunction as the most significant variables associated with poor survival. The novelty of this manuscript lies in a composition of a multivariate model that includes not only commonly used clinical covariates but also psychosocial components, so frequently omitted while looking at HF patient. The authors retrospectively evaluated 600 patients admitted between 2003 and 2006 to three Spanish hospitals for the first episode of HF. The final risk score for predicting one year mortality included age, serum creatinine and sodium levels, presence of systolic dysfunction, and dependent basic daily activities.

Even though it is also commonly known that $\mathrm{HF}$ is associated not only with impaired physical functional capacity and decreased quality of life, but also with reduced ability to deal with daily life common tasks, assessment of patients autonomy has not been included in multivariate risk models so far. It should be emphasized that taking into consideration expanding role of home-care and self-assessment, patients autonomy in daily activities as well as so- cial and family support have emerged as one of the most important factors in management of $\mathrm{HF}$. Patients with $\mathrm{HF}$, frequently elderly, may experience impairment in both basic activities of daily living (ADL), such as ability to feed oneself, as well as in instrumental ones which include those necessary for involvement in more complex and community related tasks. The relationship between impairment in ADL and HF was demonstrated recently by Bowling et al. [16] who found that impairment of instrumental ADL in patients above 65 years old, yet without HF, enrolled in the Cardiovascular Health Study is a strong, and independent predictor of HF development and mortality during long term follow up. Impairment of instrumental ADL defined as difficulty with using telephone, preparing food, performing housework, managing finances and shopping identified a group of patients at higher risk of $\mathrm{HF}$ and all-cause mortality during the following 12 -years observation. There is also data indicating that dependence for basic an instrumental ADL is strongly correlated with cognitive impairment and contributes to increased mortality in HF patients especially among elderly [17]. Social and family support is known to be related with health-related quality of life in HF patients and is considered as an important factor in adherence to planned selfcare measures in HF like weight-measurement, diuretic-adjustment, prescribed drug compliance. Wu et al. [18] have shown that a combination of medication adherence and social support, independently or in combination were significantly related to survival in HF patients. Impaired ADL and lack of social support may therefore affect compliance to prescribed medications which may consequently lead to deterioration of HF. It is hard to speculate whether this was a case in the population studied by Gomez de la Camara et al. [15]. However, the other important fact should be emphasized the population presented in a manuscript by Gomez de la Camara et al. [15], even though enrolled and followed between 2003 and 2007 was significantly undertreated. Beta-blockers were used in $31 \%$, and ACE inhibitors in $55 \%$ of patients. Similar inadherence of common clinical practice into guidelines was reported in 2003 based on EuroHeart Survey [19], however, such a low percentage of patients treated accordingly to current guidelines in period 20032006 should be worrisome. Furthermore, the studied population consisted of very high proportion of patients with preserved systolic function (75\%) therefore, whether this risk model could be applied in patients with predominantly systolic dysfunction remains questionable. 
The study by Gomez de la Camara et al. [15] stresses the need to expand our thinking on risk stratification and to treat HF patients in more holistic way taking into consideration not only his physical status but also psychosocial aspects of living. Such an approach is in line with current guidelines that recommend multidisciplinary programs which include patients' education, optimal medical treatment, psychosocial support and improved access to heathcare system [1]. Patients should be able to recognize and monitor signs and symptoms of a disease, record daily weight and increase diuretic dose and/or alert theirs doctors in case of rapid weight gain. Adherence to prescribed medical treatment and recommended diet and lifestyle are essential in HF management. Recent years brought also revolution in home monitoring of patients with implanted devices which are capable to alert healthcare providers about any changes preceding the need of hospital readmission [3, 4]. Therefore, taking into consideration novel options of HF management is seems that evaluation of psychosocial factors and impairment of daily activities may play more and more important role in $\mathrm{HF}$ population.

Conflict of interest: none declared

\section{References}

1. McMurray JJ, Adamopoulos S, Anker SD et al. ESC Guidelines for the diagnosis and treatment of acute and chronic heart failure 2012: The Task Force for the Diagnosis and Treatment of Acute and Chronic Heart Failure 2012 of the European Society of Cardiology. Developed in collaboration with the Heart Failure Association (HFA) of the ESC. Eur Heart J, 2012; 33: 1787-1847

2. Hunt SA, Abraham WT, Chin MH et al. 2009 focused update incorporated into the ACC/AHA 2005 Guidelines for the Diagnosis and Management of Heart Failure in Adults: A report of the American College of Cardiology Foundation/American Heart Association Task Force on Practice Guidelines: Developed in collaboration with the International Society for Heart and Lung Transplantation. Circulation, 2009; 119: e391-e347.

3. Cubbon RM, Gale CP, Kearney LC et al. Changing characteristics and mode of death associated with chronic heart failure caused by left ventricular systolic dysfunction: A study across therapeutic eras. Circ Heart Fail, 2011; 4: 396-403.

4. Lobodzinski S, Jadalla AA. Integrated heart failure telemonitoring system for homecare. Cardiol J, 2012; 17: 200-204.

5. Bui AL, Fonarow GC. Home monitoring for heart failure management. J Am Coll Cardiol, 2012; 59: 97-104.
6. Fonarow GC. Clinical risk prediction tools in patients hospitalized with heart failure. Rev Cardiovasc Med, 2012; 13: e14-e23.

7. Zipes DP, Camm AJ, Borggrefe M et al.; American College of Cardiology; American Heart Association Task Force; European Society of Cardiology Committee for Practice Guidelines; European Heart Rhythm Association; Heart Rhythm Society. ACC/ /AHA/ESC 2006 guidelines for management of patients with ventricular arrhythmias and the prevention of sudden cardiac death: A report of the American College of Cardiology/American Heart Association Task Force and the European Society of Cardiology Committee for Practice Guidelines (Writing Committee to Develop guidelines for management of patients with ventricular arrhythmias and the prevention of sudden cardiac death) developed in collaboration with the European Heart Rhythm Association and the Heart Rhythm Society. Europace, 2006; 8: 746-837.

8. Cygankiewicz I. Implantable cardioverter defibrillator outcome: Beyond ejection fraction? Europace, 2012 [Epub ahead of print].

9. Levy WC, Mozaffarian D, Linker DT et al. The Seattle Heart Failure Model: Prediction of survival in heart failure. Circulation, 2006; 113: 1424-1433.

10. Vazquez R, Bayes-Genis A, Cygankiewicz I et al.; MUSIC Investigators. The MUSIC Risk score: A simple method for predicting mortality in ambulatory patients with chronic heart failure. Eur Heart J, 2009; 30: 1088-1096.

11. Parkash R, Stevenson WG, Epstein LM, Maisel WH. Predicting early mortality after implantable defibrillator implantation: A clinical risk score for optimal patients selection. Am Heart J, 2006; 151: 397-403.

12. Goldenberg I, Vyas AK, Hall WJ et al.; MADIT-II Investigators. Risk stratification for primary implantation of a cardioverter-defibrillator in patients with ischemic left ventricular dysfunction. J Am Coll Cardiol, 2008; 51: 288-296.

13. Sherazi S, Zaręba W. Diastolic heart failure: Predictors of mortality. Cardiol J, 2011; 18: 222-232.

14. Meta-analysis Global Group in Chronic Heart Failure (MAGGIC). The survival of patients with heart failure with preserved or reduced left ventricular ejection fraction: An individual patient data meta-analysis. Eur Heart J, 2012; 33: 1750-1757.

15. Gómez de la Cámara A, GuerraVales JM, Magán Tapia P et al. Role of biological and non biological factors in congestive heart failure mortality: PREDICE-SCORE: A clinical prediction rule. Cardiol J, 2012; 19: 578-585.

16. Bowling $\mathrm{CB}$, Fonarow GC, Patel $\mathrm{K}$ et al. Impairment of activities of daily living and incident heart failure in community-dwelling older adults. Eur J Heart Fail, 2012; 14: 581-587.

17. Yamada S, Shimizu Y, Suzuki M, Izumi T; PTMaTCH collaborators. Functional limitations predict the risk of rehospitalization among patients with chronic heart failure. Circ J, 2012; 76: 1654-1661.

18. Wu JR, Frazier SK, Rayens MK, Lennie TA, Chung ML, Moser DK. Medication adherence, social support, and event-free survival in patients with heart failure. Health Psychol, 2012 [Epub ahead of print].

19. Komajda M, Follath F, Swedberg K et al.; Study Group on Diagnosis of the Working Group on Heart Failure of the European Society of Cardiology. The EuroHeart Failure Survey Programme: A survey on the quality of care among patients with heart failurein Europe. Part 2: Treatment. Eur Heart J, 2003; 24: 464-474. 